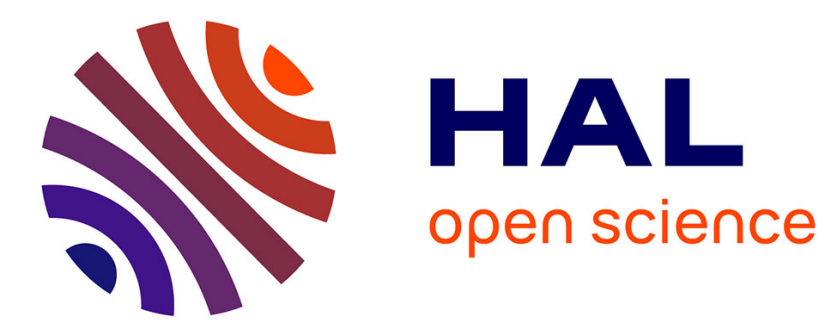

\title{
Licorne ou la guerre si nécessaire, pour maintenir ou imposer la paix \\ Jacques Aben
}

\section{To cite this version:}

Jacques Aben. Licorne ou la guerre si nécessaire, pour maintenir ou imposer la paix. Stratégique, 2017, 117, pp.255-283. hal-01979228v2

\section{HAL Id: hal-01979228 \\ https://hal.science/hal-01979228v2}

Submitted on 12 Jul 2019

HAL is a multi-disciplinary open access archive for the deposit and dissemination of scientific research documents, whether they are published or not. The documents may come from teaching and research institutions in France or abroad, or from public or private research centers.
L'archive ouverte pluridisciplinaire HAL, est destinée au dépôt et à la diffusion de documents scientifiques de niveau recherche, publiés ou non, émanant des établissements d'enseignement et de recherche français ou étrangers, des laboratoires publics ou privés.

\section{(이) $\$$}

Distributed under a Creative Commons Attribution - NonCommercial - NoDerivatives| 4.0 


\title{
Licorne ou la guerre, si nécessaire, pour maintenir ou imposer la paix
}

\author{
Jacques Aben ${ }^{1}$ \\ Professeur émérite à l'université de Montpellier \\ CRISES EA4424
}

«Nous protégeons moins nos mystères, On laisse moins faire le hasard, On se méfie du fil de l'eau, Mais c'est toujours la tendre guerre », Jacques Brel, La chanson des vieux amants. «Edgardo : M'odi e trema! Sulla tomba che rinserra, il tradito genitore, al tuo sangue eterna guerra, io giurai nel mio furore. "» ${ }^{2}$, Gaetano Donizetti, Lucia di Lamermoor, acte 1, scène 2,

Le mot «guerre» fait partie du langage commun et se trouve employé dans tous les contextes, que les dictionnaires les plus sérieux ne sont pas nécessairement d'accord entre eux : " Situation conflictuelle entre deux ou plusieurs pays, Etats, groupes sociaux, individus, avec ou sans lutte armée » (Centre national de ressources textuelles et lexicales) ${ }^{3}$ ou «Lutte armée entre groupes sociaux, et SPÉCIALEMENT entre États, considérée comme un phénomène social.» (Petit Robert). Et si l'on avait des doutes, on pourrait encore se retourner vers les origines et citer Cicéron: "Qua re non sum nescius quanto periculo vivam in tanta multitudine improborum, cum mihi uni cum omnibus improbis aeternum videam bellum esse susceptum ${ }^{4}$ (Pro Publius Sylla, http://remacle.org/bloodwolf/orateurs/sylla.htm).

Ainsi il existe maintes guerres, entre Etats ou pas, avec armes ou pas. Alors comment appeler une situation comportant la possibilité d'emploi de la force armée et se soldant par des pertes humaines et matérielles? C'est la question que l'on peut se poser dans un cas particulier, celui de la plus récente crise en Côte d'Ivoire et de l'opération Licorne, qui a amené un corps expéditionnaire français sur place, avec le mandat d'assurer la sécurité des expatriés et maintenir la paix, par l'emploi de la force armée si nécessaire.

$\mathrm{Au}$ fond, cette question reste tout à fait documentaire, sinon oiseuse, tant que la qualification des faits n'emporte pas de conséquence pour les parties prenantes ou pour des tiers concernés : le fait de refuser l'emploi de l'expression " guerre économique » à des chercheurs ou des journalistes n'est sûrement pas le bon moyen de s'exonérer de l'indemnisation des chômeurs qui en sont les victimes, ou d'empêcher la poursuite des auteurs de délits ou crimes que cette « guerre » occasionnerait. A l'inverse, l'existence d'un « droit de la guerre » permet d'autoriser ou d'interdire telle ou telle action, et finalement de poursuivre les auteurs de transgressions, pourvu qu'il existe une cour, nationale ou internationale selon le cas, à cet effet $^{5}$. On peut s'attendre alors à ce que ce droit donne une définition des circonstances dans lesquelles il pourra s'appliquer et c'est bien ce qui a été fait par les conventions successives sur le droit de la guerre, comme le montrent les citations ci-après :

«(...) tout en recherchant les moyens de sauvegarder la paix et de prévenir les conflits armés entre les nations, il importe de se préoccuper également du cas où l'appel aux armes serait amené

\footnotetext{
${ }^{1}$ La rédaction de ce texte a bénéficié des remarques judicieuses du général d'armée (2s) Elrick Irastorza, ancien commandant de Licorne. Il est bien évident qu'il ne porte aucune responsabilité sur les erreurs ou fautes qui pourraient demeurer.

«Écoutez-moi et tremblez ! Sur la tombe de mon père outragé, et dans ma colère, j'ai juré guerre éternelle à votre sang ! »

${ }_{3}^{3}$ « De l'a. b. frq. *werra « troubles, désordres; querelle », cf. l'a.h. all. werra « scandale; querelle », m. néerl. werre « confusion, désordre; querelle, guerre ». Attesté en lat. médiév. au ix ${ }^{\mathrm{e}} \mathrm{s}$. (Du Cange; Nierm.), *werra a éliminé le lat. class. bellum...».

${ }^{4}$ «Je n'ignore donc pas à quels périls je suis exposé au milieu d'une si grande foule d'hommes pervers; car je vois que j'aurai à soutenir, seul contre tous les méchants, une guerre éternelle. »

${ }^{5}$ La perte progressive d'assise de la Cour pénale internationale n'est sans doute pas de bon augure à cet égard.
} 
par des événements que Leur sollicitude n'aurait pu détourner. », préambule de la Convention concernant les lois et coutumes de la guerre sur terre, conclue à La Haye, le 29 juillet 1899.

« (...) la présente Convention s'appliquera en cas de guerre déclarée ou de tout autre conflit armé surgissant entre deux ou plusieurs des Hautes Parties contractantes, même si l'état de guerre n'est pas reconnu par l'une d'elles. », conventions de Genève du 12 août 1949, article 2 commun.

«(...) tous les conflits armés (...) qui se déroulent sur le territoire d'une Haute Partie contractante entre ses forces armées et des forces armées dissidentes ou des groupes armés organisés qui, sous la conduite d'un commandement responsable, exercent sur une partie de son territoire un contrôle tel qu'il leur permette de mener des opérations militaires continues et concertées et d'appliquer le présent Protocole. » $2^{\text {ème }}$ protocole additionnel aux conventions de Genève du 12 août 1949.

Ces textes fondamentaux donnent l'impression, eux aussi, d'hésiter devant l'emploi du mot " guerre ». D'un côté, on " déclare » formellement la guerre ${ }^{6}$, alors que l'on ne déclare pas un « conflit armé » et, de l'autre côté, on peut avoir un « état de guerre » qui comprend aussi les cas «d'appel aux armes » ne ressortissant pas au «droit des conflits armés ». D'une certaine manière, ce second usage du mot guerre, par ceux-là mêmes qui étaient habilités à la déclarer, semble grandement simplifier les débats, puisque applicable, au minimum, à tout type de conflit armé, y compris lorsqu'il «n'est pas reconnu » par l'un des protagonistes.

La question qui sera examinée dans ce qui suit est celle de l'emploi du mot "guerre » dans le cas de la crise qui a déchiré la Côte d'Ivoire entre 2002 et 2011 et de l'intervention du corps expéditionnaire «Licorne » qui en a été la conséquence directe. Mais avant d'entrer dans les détails, une présentation générale s'impose.

L'opération Licorne en Côte d'Ivoire a été déclenchée le 22 septembre 2002, même si son premier chef, le général Emmanuel Beth, n'a pris officiellement ses fonctions sur place que le $1^{\text {er }}$ octobre, accompagné par l'état-major tactique ${ }^{7}$ de la $11^{\text {ème }}$ brigade parachutiste. Elle a été close le 21 janvier 2015, et les troupes encore présentes en Côte d'Ivoire (580 militaires) sont passées sous le statut de Forces françaises en Côte d'Ivoire, forces de présence «pour assurer un relai opérationnel sur une zone d'intérêt stratégique ${ }^{8}$ comme le prévoit l'accord de défense signé par ce pays et la France le 26 janvier 2012.

Cette opération aura donc duré exactement 13 ans et 4 mois, ce qui est sans doute long, mais pas plus que l'opération Trident (15 ans) et a fortiori Epervier (28 ans).

La crise qui a été à l'origine de cette opération était prévisible. Avec une taille moyenne à l'échelle africaine : $332000 \mathrm{~km}^{2}$ et 23 millions d'habitants, la Côte d'Ivoire a été longtemps un exemple pour le reste du continent africain : en paix avec ses voisins ; une administration efficace; une économie prospère et l'amitié protectrice des Etats Unis autant que de la France. Félix Houphouët-Boigny, fondateur et premier président de la République de Côte d'Ivoire, avait pris avantage de la Guerre froide pour devenir l'interlocuteur privilégié des puissances occidentales et, ainsi, un acteur influent sur la scène internationale.

\footnotetext{
${ }^{6}$ Article 35 de la Constitution du 4 octobre 1958 : « La déclaration de guerre est autorisée par le Parlement».

${ }^{7}$ Un état-major tactique assure la coordination au sol avec l'environnement immédiat et gère les problèmes annexes à l'opération (http://www.penseemiliterre.fr/pourquoi-projeter-un-chef-supplementaire_2015006.html). $8 \mathrm{http} / /$ www.defense.gouv.fr/ema/forces-prepositionnees/cote-d-ivoire/dossier/les-forces-francaises-en-cote-divoire
} 
Les signes avant-coureurs de la crise sont apparus dans les années 1980 avec l'effondrement du secteur primaire, pétrolier autant qu'agricole. Son effet politique a été aggravé par un contexte de corruption et d'extrêmes inégalités : $31 \%$ de la population au dessous du seuil de pauvreté en 1993.

Une autre racine de la crise se trouve dans les conflits ethniques, non seulement entre tribus, comme il est commun en Afrique, mais aussi entre Ivoiriens « vrais » et Ivoiriens immigrés des pays voisins; entre le sud et le nord; entre chrétiens et musulmans. Cette tendance spontanée a été aggravée par une instrumentalisation à des fins politiciennes par une presse locale assez éloignée des bonnes pratiques journalistiques. Elle a même été érigée en règle juridique avec le concept d'ivoirité ${ }^{9}$, qui a permis d'écarter l'ancien premier ministre Alassane Dramane Ouattara de l'élection présidentielle du 22 octobre 2000.

Mais les signes avant-coureurs les plus dangereux ont été les mutineries successives de militaires : 1990, 1992, 1999, 2001 et finalement 2002. Les revendications présentées par les mutins étaient généralement économiques : conscrits demandant à rester dans l'armée ou professionnels revendiquant la même limite d'âge que les fonctionnaires. Seule la mutinerie de 1999 a pris la tournure d'un coup d'État, le général Robert Gueï, chef d'état-major des armées, s'emparant du pouvoir détenu par le président Henri Konan Bédié, mais organisant curieusement des élections en 2000, gagnées par Laurent Gbagbo, l'opposant de toujours.

\section{1 - Une guerre civile en Côte d'Ivoire}

Le 19 septembre 2002 à 3 heures du matin, des hommes lourdement armés ont attaqué des points stratégiques simultanément à Abidjan et Bouaké, les deux plus grandes villes de Côte d'Ivoire ainsi qu'à Korhogo, grande ville du nord, l'un des fiefs de l'opposition au président Laurent Gbagbo. Abidjan est reprise dans le courant de la matinée par les forces loyalistes et on a pu croire, un temps, que ce coup de force était un échec. La réalité était plus prosaïque et moins glorieuse : les forces loyalistes n'avaient pas encore contrattaqué à Bouaké et Korhogo, laissant aux assaillants le temps de se renforcer avec les armes prises dans les casernes de ces villes et d'enrôler de nouvelles recrues. En deux jours on dénombrerait au moins 270 morts et 300 blessés, rien qu'à Abidjan.

Jusqu'à la fin septembre 2002, les observateurs semblent s'être perdus en conjecture sur la nature exacte des évènements en cours. Le 19 septembre, le président Gbagbo affirmait à la télévision que les attaquants utilisaient des «armes lourdes, nouvelles pour la plupart, des armes dont ne dispose pas notre armée - donc on ne peut pas dire que c'est l'armée de Côte d'Ivoire qui se rebelle - des armes utilisées dans les armées étrangères ou achetées à des gouvernements ». Plus clairement c'est le Burkina Faso, puis le Libéria, le Mali et la Sierra Leone, qui ont été désignés, à mots plus ou moins couverts, comme agresseurs. En conséquence, le ministre de la défense, Moïse Lida Kouassi, décrétait Bouaké et Korhogo «zones de guerre » et déclarait à la presse : "Il ne fait aucun doute que la Côte d'Ivoire est confrontée à une agression armée dont l'objectif est de renverser les institutions légales. (...) Il ne peut donc s'agir que d'une agression extérieure ${ }^{10}$. Une telle affirmation, si elle avait donné lieu à une demande d'appui militaire à la France et si elle avait été prise au sérieux par celle-ci, l'aurait peut-être conduite à se ranger franchement à côté des forces loyalistes, au nom de l'accord de défense du 24 août 1961 liant les deux pays.

\footnotetext{
${ }^{9}$ Loi 94-642, du 13 décembre 1994, article 49 : « (...) personne ne peut être élu Président de la République (...) s'il n'est pas né ivoirien, de parents eux-mêmes ivoiriens. »

${ }^{10}$ Le Monde 21 et 28 septembre 2002.
} 
Pourtant, les assaillants se présentaient eux-mêmes comme des mutins ${ }^{11}$, même s'ils pouvaient avoir bénéficié du soutien d'Etats voisins : "Ce que nous voulons c'est que nos camarades qui doivent être démobilisés en décembre restent dans l'armée ${ }^{12}$. Une raison bien maigre pour déclencher une insurrection armée, sauf si elle n'est que la goutte d'eau de trop dans un océan de mécontentement lié à une politique xénophobe conduite résolument sous l'autorité du président Gbagbo. On en trouve un signe, sinon une preuve, dans le fait que très peu après la prise de Bouaké par les insurgés, les habitants baoulés et dioulas de cette ville descendaient dans la rue chaque jour pour conspuer le régime légal : "On veut pas Gbagbo!». Et comme pour leur donner raison, un journaliste de la Radio-télévision ivoirienne (RTI) pouvait affirmer sans crainte dès le 6 octobre : "Pourquoi la Côte d'Ivoire, qui est en temps de guerre, ne mettrait pas dehors ceux qui sont principalement à la base de nos malheurs? Il suffirait tout simplement d'expulser vers le Faso ne serait-ce que 500000 Burkinabè $»^{13}$. D'ailleurs, dès la nuit suivante, les Forces armées de Côte d'Ivoire (FANCI), qui avaient repris (provisoirement) Sakassou dans la journée, ont tenté de reprendre Bouaké, à $40 \mathrm{~km}$ au nord-est. Mais elles se sont heurtées à une résistance suffisamment résolue pour devoir renoncer après moins d'une journée de combat et refluer vers Abidjan. En revanche elles se sont avérées plus efficaces à Daloa, $180 \mathrm{~km}$ au sud-ouest de Bouaké, qu'elles ont pu reprendre après trois jours de combats acharnés, entre le 14 et le 17 octobre. Et pour confirmer une nouvelle fois le caractère inter-ethnique de la guerre civile, les troupes loyalistes ont commis nombre d'exactions à l'issue des combats : brutalités, viols, exécutions sommaires, massacres, comme s'il était nécessaire de «venger» les victimes des mêmes exactions commises par les rebelles un mois plus tôt.

Pourtant c'est ce même 17 octobre qu'une première trêve est conclue entre les rebelles et le gouvernement légal. Cette sorte de parité reconnue par la communauté internationale donnait une crédibilité aux rebelles et, d'une certaine manière, les conduisait à se muer en groupe politique : le Mouvement patriotique de Côte d'Ivoire ou MPCI. Mais comme aurait pu dire la Cassandre de Giraudoux, «c'était la première des trêves, la suivante l'attend ».

En effet, il ne faudra pas plus d'un mois avant que les troupes loyalistes repartent à l'assaut dans l'ouest, vers les villes de Man et Vavoua. Ce choix n'est pas anodin si l'on sait que ces villes sont les verrous nord-ouest de la «boucle du cacao $»^{14}$, et que Man est le centre de la deuxième zone caféière de Côte d'Ivoire. La prise de ces villes signifiait donc la sécurisation des principales richesses agricoles du pays au profit du gouvernement légal.

C'était sans compter sur le capital d'hostilité réuni alors par Laurent Gbagbo et son ethnie, les Bétés. En effet au même moment où ses troupes tentaient de repousser vers le nord les combattants du MPCI, deux nouveaux mouvements à connotation ethnique prenaient les armes contre elles: le Mouvement populaire ivoirien du grand ouest (MPIGO) et le Mouvement pour la justice et la paix (MJP). Le MPIGO n'était en fait que la façade politicomilitaire de la tribu Yacouba ou Dan, voulant venger l'un des siens, le général Guéï, assassiné par des affidés de Laurent Gbagbo le 19 septembre.

L'année 2002 se terminait donc par deux échecs pour le gouvernement légal. D'une part son offensive vers le nord était enrayée puisque Danane et plus tard Man étaient reprises par les insurgés, malgré son absolue supériorité aérienne. D'autre part, ces mêmes insurgés convenaient, le 23 décembre, de former un mouvement unique : les Forces nouvelles, avec leurs propres forces armées, les FAFN. C'est ainsi qu'une nouvelle trêve pouvait être acceptée le 14 janvier par l'ensemble de la rébellion d'un côté, le gouvernement légal de

\footnotetext{
${ }^{11}$ Le Monde 20 septembre 2002.

${ }^{12}$ Un mutin cité par Le Monde, 23 septembre 2002.

${ }^{13}$ Le Monde,

${ }^{14}$ C. Benveniste, La boucle du cacao en Côte d'Ivoire, étude régionale des circuits de transport, ORSTOM, Paris, 1974.
} 
l'autre côté, marquant à nouveau l'équilibre établi par la communauté internationale entre un gouvernement légal et une rébellion. Marc Le Pape et Claudine Vidal pouvaient alors titrer dans Le Monde : «L'état de guerre s'installe en Côte d'Ivoire » ${ }^{15}$, reprenant ainsi l'expression même de l'article 2 commun des conventions de 1949.

Le début de l'année 2003 ne devait pas décevoir ces deux experts, en révélant l'un des côtés les plus sombres de cette guerre, l'ingérence de bandes criminelles venues du Liberia et mettant à feu et à sang toute la région sud-ouest du pays. Certes, chacun des deux camps ivoiriens a pu, à son tour, aller chercher des mercenaires ou simplement des alliés dans ce pays qui sortait à peine de vingt ans de guerre civile. Mais les bandes dont il s'agit ont semblé simplement vouloir mettre la région de Toulepleu en coupe réglée, au point qu'une alliance se formera entre FANCI, FAFN et Licorne pour les renvoyer au Liberia par la force, mais en laissant derrière elles de très nombreuses victimes d'exactions.

Les accrochages entre forces militaires et les agressions contre les civils ont continué de s'égrener tout au long de l'année 2003 et au début de l'année 2004. Il serait fastidieux et sans profit d'en faire la narration, une liste suffit : 28 janvier, combats à Agboville $(80 \mathrm{~km}$ au nord d'Abidjan) entre Abbeys et Dioulas, «Toute la nuit, il y a eu des appels à la guerre. Tous les commerces dioulas et le marché ont été attaqués »; 10 mars, combats à Bangolo (ouest), au moins 60 morts chez les civils ; 18 avril, attaque d'hélicoptère à Vaoua (centre) plusieurs morts ; 12 décembre, échange de coups de feux à Abidjan entre des gendarmes et des inconnus, au moins 12 morts...

En revanche il importe de s'arrêter sur la manifestation de l'opposition réunie (G-7) du 25 mars 2004. Toute manifestation était interdite par les autorités jusqu'au 30 avril à la suite de divers débordements intervenus au cours du mois de mars. Les responsables internationaux en poste en Côte d'Ivoire avaient demandé aux leaders de l'opposition de surseoir à leur projet, et aux autorités d'ouvrir un dialogue avec l'opposition. Malgré cela, la manifestation a eu lieu; elle a été dispersée avec une rare violence par les forces de l'ordre et par des milices rappelées spécialement à Abidjan. La répression a continué pendant les trois jours suivants, y compris dans les hôpitaux et au domicile d'opposants fichés. Finalement le compte des tués s'élèverait selon l'ONU à 120, mais pour les journalistes français ayant couvert les évènements, ce serait plutôt «plusieurs centaines ». Surtout le rapport de l'ONU note : «ce qui s'est passé le 25 mars et les jours suivants a été une tuerie aveugle de civils innocents de la part des forces de l'ordre. (...) il a été démontré qu'il y avait dans ce massacre des populations ciblées, parmi lesquelles des communautés du nord du pays et celles de pays voisins (...) qui ont fait l'objet de grandes violations de droits de l'homme, exécutions sommaires et extrajudiciaires, torture, détentions arbitraires, disparitions [et viols, évidemment $\left.{ }^{16}\right]$ sans lien direct avec la marche ${ }^{17}$.

On pourrait dire que l'année 2004 s'est terminée un peu comme elle avait commencé, au sens où, malgré le cessez-le-feu, les FANCI on à nouveau tenté de repousser les FAFN vers le nord. C'est ainsi que des chasseurs-bombardiers Sukhoï 25 pilotés par des mercenaires biélorusses ${ }^{18}$ sont venus bombarder et mitrailler des positions FAFN, notamment à Bouaké, les 4 et 5 novembre, alors que des colonnes terrestres tentaient, sans succès, de reprendre Sakassou et Bouaké.

\footnotetext{
${ }^{15} 14$ janvier 2003.

${ }^{16}$ «Le viol comme arme de guerre. Rapport de l'Unesco », Confluences Méditerranée, 1/2008 (N64), p. 99104.

${ }^{17}$ Haut Commissariat des Nations Unies aux droits de l'homme, Rapport de la Commission d'enquête sur les événements liés à la marche prévue le 25 mars 2004 à Abidjan (Côte d'Ivoire), Genève, 29 avril 2004, p.9. L'un des journalistes en question a été exfiltré par sa rédaction deux jours après, celle-ci craignant pour sa vie.

${ }^{18}$ Et peut-être guidés par des contrôleurs aériens israéliens.
} 
Entre 2005 et 2010 la situation ira en s'accalmissant, même si ici et là des combats auront lieu et des exactions seront commises. Tellement qu'enfin des élections présidentielles promises depuis longtemps pourront avoir lieu les 31 octobre et 28 novembre - sans la clause d'ivoirité, donc avec la candidature d'Alassane Ouattara, soutenu sans surprise par les Forces nouvelles. Mais on sait quelle rocambolesque situation en est résultée, avec deux candidats déclarés élus par deux instances étatiques distinctes, et la tentative de Laurent Gbagbo de garder le pouvoir par la force. Et c'est bien la force qui a permis de trancher ce nœud gordien, mais celle d'Alassane Ouattara, les FAFN finissant par prendre le dessus sur les FANCI et les milices pro-Gbagbo, après une bataille de quatre mois.

Ainsi il a bien existé une guerre civile pendant neuf années en Côte d'Ivoire, entre le pouvoir légal et les populations du sud d'un côté et celles du nord et de l'ouest de l'autre. Mais l'évidence qui résulte de la longue liste des combats ne peut prendre tout son sens qu'après examen des pertes : comme le note le Stockholm International Peace Research Institute (SIPRI), on ne peut parler de conflit armé majeur que si l'on compte au moins 1000 tués en une seule année.

A priori il existe une source dédiée et théoriquement digne de confiance avec la base de données sur les conflits de l'université d'Uppsala (UCDP). Pour la période 2002-2011, cette base affiche 2576 morts $^{19}$. Pour ceux qui ont eu une petite connaissance expérimentale de ce conflit et de la violence qui peut se déclencher dans ces régions, ce chiffre paraît bien faible : «(...) le bataillon antiémeute (BEA), a semé la terreur. Il s'agissait de 'nettoyer' la ville des supposés complices des assaillants du Nord. (...) Pendant deux jours, rafles et rapts ont continué. Le BEA a opéré une 'descente' dans un quartier d'immigrés, Orly 2, faisant dixneuf morts. C'est alors, dimanche, qu'une foule de musulmans, Ivoiriens du Nord et étrangers confondus, s'est massée autour de la grande mosquée, aux cris de 'Voici nos corps, venez nous tuer tous, pour en finir' $\gg{ }^{20}$.

Cette impression est corroborée par l'examen de diverses autres sources. L'université de Sherbrooke (Canada) annonce pour sa part «plus de $3000 »$, mais pour la seule sous-période 2000-2005, ce qui est encore conservateur par rapport aux "plusieurs milliers de morts » décomptés par Le Monde ${ }^{21}$ pour la seule période 2002-2003. Or le rapport rédigé pour le seul conflit post-électoral (2010-2011) par la Commission d'enquête nationale ivoirienne, et avalisé par le Conseil de sécurité de l'ONU, parvient à un total de $3248^{22}$, soit un total minimum de 6500 pour l'ensemble de la crise. Autre source, partielle celle-ci, la base établie par l'ONG «Coalition pour la justice et le pardon des victimes de guerre de Côte d'Ivoire » qui, pour la seule région du Haut-Sassandra et sur l'ensemble de la guerre, décompte 9736 décès, auxquels il faut ajouter 711 disparus. Il n'est évidemment pas question d'extrapoler ce chiffre sur les 31 régions du pays, même sur une base très conservatrice, mais si l'on considère qu'il y a eu au moins quatre zones où on eu lieu des combats importants : le sud-ouest, Abidjan et sa région, Bouaké et sa région et Korhogo, des chiffres supérieurs à 10000 ne sembleraient pas si incroyables.

Comme toujours, la vérité doit être quelque part entre les extrêmes, mais elle est déjà effrayante, d'autant que les protagonistes, mais surtout le pouvoir légal, ont tout fait pour

\footnotetext{
${ }^{19} \mathrm{http}: / /$ ucdp.uu.se/\#country/437

${ }^{20}$ Le Monde, 28 octobre 2002.

${ }^{21} 24$ juin 2004.

${ }^{22}$ Rapport au Conseil de sécurité de la Commission d'enquête internationale indépendante sur la Côte d'Ivoire, A/HRC/17/48, p.2.
} 
museler les médias étrangers, susceptibles d'en rendre compte ${ }^{23}$. Elle l'est a fortiori si l'on considère comment ces décès sont intervenus. Certes, les militaires se sont battus avec des armes lourdes et ont certainement supporté des pertes significatives, mais ce sont les civils, et des militaires prisonniers, qui ont payé le plus lourd tribut et souvent d'une manière qui provoque plus l'horreur que l'effroi. Toutes les organisations, qu'elles soient internationales ou non gouvernementales ont rendu compte des atrocités commises par les deux parties, pour qui le " droit de la guerre » s'est avéré un concept vide de sens. Cela ressortait abondamment du rapport de l'ONU déjà cité sur la répression de la manifestation du 25 mars 2004, mais cela ressort aussi du rapport d'Amnesty International sur le massacre de gendarmes et de leurs enfants à Bouaké en 2002 :

"J'ai été arrêté avec trois de mes enfants, l'un âgé de vingt et un an, a été tué sur le coup le dimanche soir. Mes deux autres fils, âgés de dix-neuf ans et de vingt- trois ans, ont été blessés. Je suis resté à côté d'eux tout le lundi mais ils sont morts ce jour-là des suites de leurs blessures. » ${ }^{24}$

Si l'on revient vers la base de données de l'UCDP, on dispose d'une distribution des décès sur l'ensemble de la période. Elle fait apparaître deux pics, en 2002 et 2011, avec respectivement 998 et 467 morts. En d'autres termes, dès la première année, ce conflit armé entrait dans le groupe des «majeurs » au sens du SIPRI, de sorte que l'emploi de l'appellation « guerre » à son propos n'a rien d'excessif.

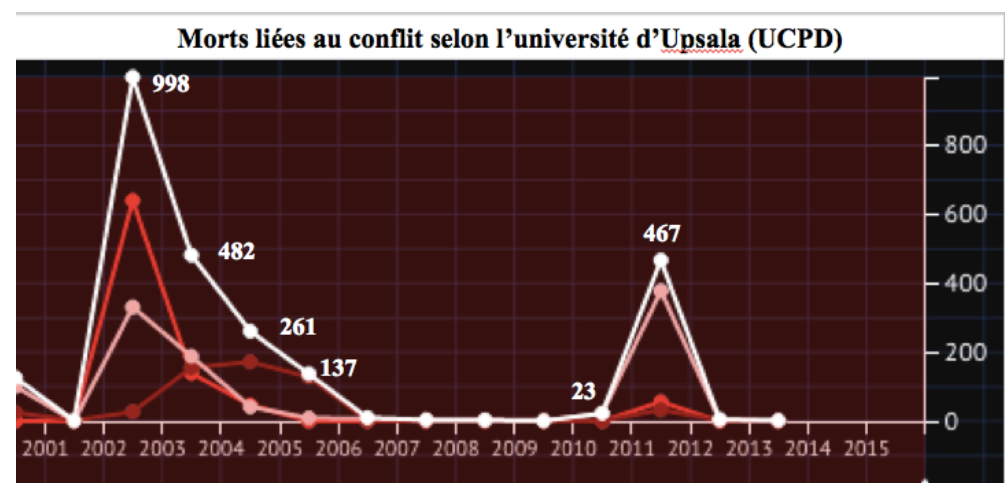

\section{2 - Une économie de guerre}

L'image que l'on se fait spontanément de l'économie de guerre, est évidemment celle de la rareté, de la disette (économie de «guère »?). La guerre civile en Côte d'Ivoire ne fait pas exception. Selon l'ONU, il y avait de 600000 à 750000 déplacés à la fin de 2002 et 250000 réfugiés dans les pays voisins ${ }^{25}$. Rien qu'à Bouaké, 250000 personnes avaient quitté la ville dès les premiers jours, dont beaucoup de commerçants qui «ont baissé leurs rideaux, déserté Bouaké ou attendent chez eux des lendemains meilleurs ${ }^{26}$. Ce genre de situation s'est répété dans tous les lieux où des combats ont eu lieu, comme Korhogo, Man, Daloa, Duékoué, Agboville, Danané et évidemment Abidjan, de sorte que ce sont au moins six à huit millions d'Ivoiriens qui ont été frappés par la disette à un moment ou à un autre de cette guerre : " ' $J$ ' $a i$ une vingtaine de personnes réfugiées chez moi dont trois femmes qui allaitent, et je n'ai rien à leur donner', dit Claudine, une jeune enseignante. 'Je n'avais plus que deux oufs ; j'en ai

\footnotetext{
${ }^{23}$ «Les événements du 19 septembre 2002 ont plongé la Côte d'Ivoire dans la période la plus troublée de son histoire. Pour la presse, les coups viennent de toutes parts, des forces armées comme des rebelles. ", Reporters sans frontières, rapport annuel 2003, http://archives.rsf.org/article.php3?id_article $=6303$

${ }^{24}$ Amnesty International, Côte d'Ivoire une série de crimes impunis, 27 février 2003, p.11.

${ }^{25} \mathrm{http}: / /$ www.un.org/fr/africarenewal/vol17no2/172cifr.htm et Le Monde du 24 juin 2004.

${ }^{26}$ Le Monde, 11 octobre 2002.
} 
donné un aujourd'hui à ma fille de 7 ans, elle aura l'autre demain. Nous, les adultes, cela fait deux jours qu'on n'a rien mangé', se plaint, de son côté, une femme d'âge mur $»^{27}$.

Mais même si les magasins avaient été ouverts, encore aurait-il fallu que les marchandises leur parviennent. Or, notamment depuis la fameuse affaire Firmin Mahér ${ }^{28}$, on sait que l'économie de la Côte d'Ivoire a été gangrenée par le rançonnement des transports routiers mais aussi des taxis, aussi bien par les «corps habillés » du sud, que par les guérilleros du nord $^{29}$. Le résultat, c'est que des déplacements qui prenaient normalement quelques heures en coûtaient dorénavant plusieurs dizaines, sans compter les bakchichs.

Le transport ferroviaire aussi a été mis en péril par la guerre et la partition du pays, non pas par des destructions de lignes mais par l'impossibilité pour les deux protagonistes de s'entendre sur le contrôle du trafic, sachant que la ligne principale exploitée par la SITARAIL, qui appartient au groupe Bolloré, va de Ouagadougou, au Burkina Faso, à Abidjan, en passant par Ferkessédougou, à l'est de Korhogo, et par Bouaké ${ }^{30}$.

Mais cette gangrène particulière est loin d'être la seule car l'économie de guerre engendre des situations de rente pour ceux qui disposent du pouvoir de protéger ou d'aider: " $A u$ sud la rente issue de la filière cacao est siphonnée par des proches du pouvoir à travers un certain nombre d'institutions censées 'aider' les planteurs. Au nord, des membres éminents des Forces nouvelles siphonnent la rente cotonnière. Ces prélèvements entretiennent une économie parallèle qui se nourrit du trafic d'armes, du blanchiment d'argent. A des niveaux plus bas de la hiérarchie prédatrice se situent les milices (...) et les forces de sécurité qui trouvent un supplément de revenu en dressant des barrages routiers à travers tout le pays pour arrêter et racketter les civils. $\rangle^{31}$

Avec l'instauration d'un couvre-feu nocturne à Abidjan, les entreprises se sont trouvées face au risque de chômage technique. On a vu des situations où des ouvriers faisant le quart $0 \mathrm{~h}-8 \mathrm{~h}$ devaient embaucher dès $19 \mathrm{~h}$; où ceux qui le pouvaient dormaient sur leur lieu de travail, comme les boulangers, les journalistes ou les chauffeurs de bus de la SOTRA; où les plus chanceux étaient payés à plein temps en travaillant à mi-temps; où les moins chanceux étaient licenciés.

A cela il faut ajouter la fermeture de la plupart des agences bancaires du pays, soit parce qu'elles avaient été pillées - y compris par des soldats de Licorne chargés de les garder ${ }^{32}$ soit parce que leurs employés ne pouvaient plus s'y rendre.

La combinaison de ces différents désordres a abouti à des situations de blocage à de plus hauts niveaux. Là où il fallait une semaine pour décharger un navire transportant du riz, il en fallait deux pendant le couvre-feu. Au printemps 2004, la société Nestlé souhaitait relancer la production de café autour de Man. Voulant redorer son blason, elle proposait un système de «commerce équitable» en payant les producteurs sensiblement au dessus du «prix de guerre » pratiqué par les intermédiaires libanais, et même en payant «l'impôt de guerre »aux FAFN. Mais, en contrepartie, Nestlé voulait la réouverture des guichets bancaires de Man, pour que ses employés n'aient pas à transporter des espèces, et une protection de ses camions pour éviter le racket sur les routes « contrôlées » par les FANCI. Autant dire que le projet n'a pas abouti, du moins à ce moment-là.

\footnotetext{
${ }^{27}$ Ibidem

${ }^{28}$ Meurtre d'un « coupeur de route » en 2005 par des soldats de la force Licorne, convaincus, à tort ou à raison, qu'ils en avaient reçu l'ordre de tous les échelons de la hiérarchie, jusqu'au général commandant la force.

${ }^{29}$ Il était de notoriété publique que les militaires des FANCI payaient les employés de leur direction des ressources humaines pour être affectés dans les zones où le racket routier était le plus juteux.

${ }^{30}$ C'est une situation qui rappelle étrangement celle du Kosovo, où la rivière Ibar, qui sépare Serbes et Albanais, introduit une solution de continuité sur la ligne Belgrade-Skopje

${ }^{31} \mathrm{http}: / /$ conte.u-bordeaux4.fr/Edito/editoci4.htm

${ }^{32}$ En 2003 à Bouaké et en 2004 à Man, http://www.liberation.fr/planete/2004/01/03/soldats-francais-accuses-devol-en-cote-d-ivoire_463975 et http://www.afrik.com/article8540.html
} 
On imagine que les désordres ayant touché la Côte d'Ivoire n'ont pas été sans conséquence sur l'environnement de ce pays. Par exemple, le blocage de la circulation ferroviaire a eu des conséquences désastreuses pour le Burkina Faso, pays enclavé et très pauvre : $70 \%$ des importations maritimes de marchandises générales et $25 \%$ des approvisionnements en hydrocarbures transitaient par la Côte d'Ivoire avant la crise. C'est encore pire pour le Mali, avec respectivement $75 \%$ et $85 \%$. Pour l'Union Economique et Monétaire Ouest-africaine (UEOMOA), la Côte d'Ivoire fait figure de pilier, avec environ 40\% du PIB total, 59,4 \% des exportations et $42,1 \%$ des importations ${ }^{33}$. Le FMI pouvait donc légitimement s'inquiéter : «La reprise économique que connaît actuellement l'Afrique de l'Ouest est menacée par la crise politique qui persiste en Côte d'Ivoire. L'impact économique de la contestation des résultats des élections de l'an dernier sur l'Union économique et monétaire ouest-africaine (UEMOA) et sur l'ensemble de la région va en s'aggravant. ${ }^{34}$

L'exemple le plus frappant de dépendance économique à l'égard de la guerre en Côte d'Ivoire est celui du cacao, dont on a vu plus haut à quel point il préoccupait les deux parties. En effet la Côte d'Ivoire se trouve être le plus gros producteur mondial de cette matière première, avec $31,6 \%$ du total aujourd'hui, loin devant le Ghana $(18,2 \%)^{35}$, de sorte que les variations de sa production ont toutes chances d'influencer significativement les cours mondiaux. Le résultat, c'est qu'au cours du premier mois de guerre, ces cours ont bondi de $10 \%$ : mauvaise nouvelle pour tous les producteurs de chocolat mais bonne nouvelle pour l'économie ivoirienne. Comme l'on pouvait s'y attendre la conclusion de la première trêve, le 17 octobre a eu l'effet inverse, avant que les incertitudes sur la robustesse du cessez-le-feu renvoient les prix à la hausse.

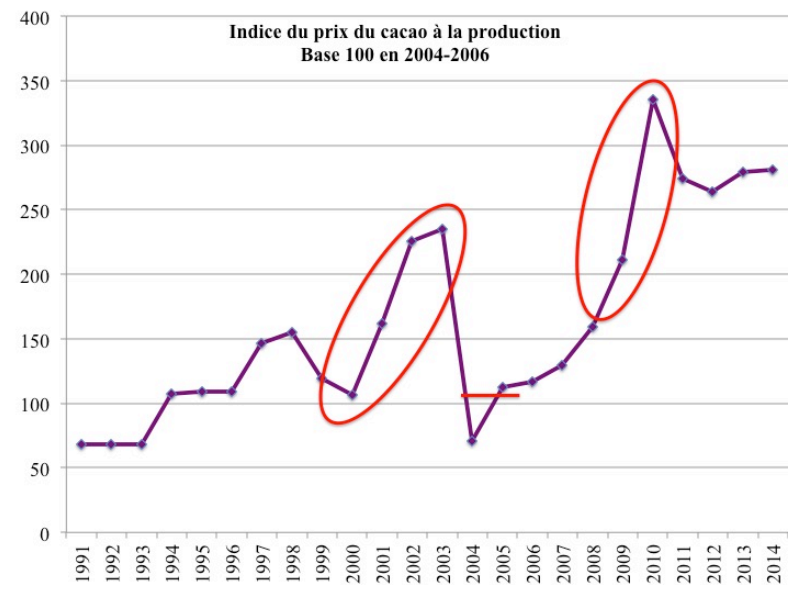

Si l'on considère l'évolution des cours sur l'ensemble de la période de guerre, on observe une tendance globale à la hausse, qui se perpétue d'ailleurs jusqu'à aujourd'hui, avec un triplement entre 2006 et 2016, et 10\% entre 2015 et 2016. C'est la traduction de la progression du revenu mondial et de la place du chocolat dans les préférences des consommateurs aisés. Autour de cette tendance, on aperçoit des fluctuations avec deux pics significatifs en 2002-2003 d'une part, en 2010 d'autre part. En d'autres termes, et sans grande surprise, les poussées des cours dessinent les poussées de la violence ou de l'anticipation de la violence en Côte d'Ivoire, telles qu'elles apparaissaient dans le décompte des pertes.

Des schémas semblables sont visibles sur les séries des grandes variables macroéconomiques. Comme l'on pouvait s'y attendre, le taux de croissance du PIB en volume connaît une chute

\footnotetext{
${ }^{33}$ Bio G. Soulé, « L’impact de la crise ivoirienne sur le commerce régional », Politique africaine, 2003/1 (

${ }^{34}$ Bulletin en ligne du 17 mars 2011, www.imf.org (consulté le 03/12/16)

${ }^{35}$ faostat3.fao.org. (consulté le 03/12/16)
} 
nette en 2002 et 2003 au point de devenir négatif et c'est bien pire en 2011, témoignant du fait qu'au cours de ces quatre mois fatidiques la violence n'a plus été contrôlée, contrairement à ce que pourraient laisser penser les chiffres de pertes de l'UCDP (Gbagbo ne disait-il pas qu'il voulait « braiser » Abidjan ?).

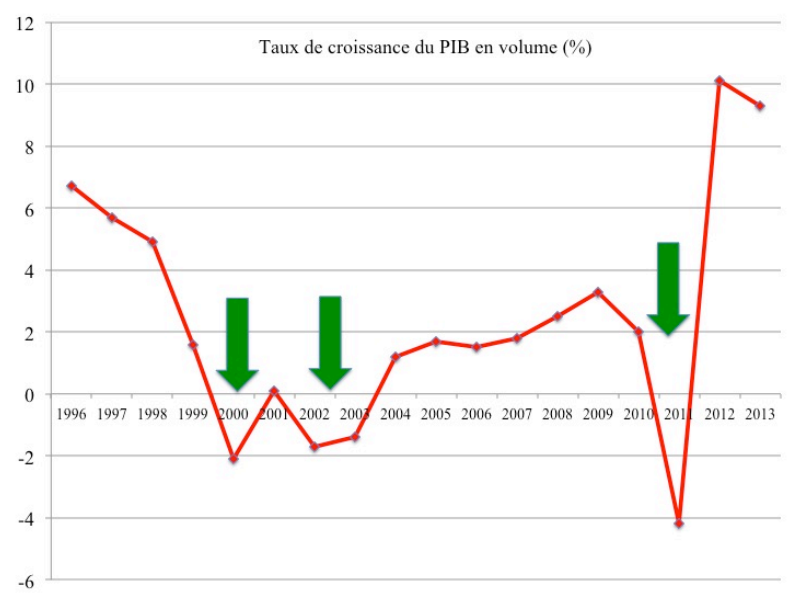

En matière de conflits armés, on parle souvent d'inflation «obsidionale », à propos d'une hausse des prix résultant de l'impossibilité de s'approvisionner du fait du siège que subit une citée ou tout territoire qui connaît la guerre. Si l'on n'y prend pas garde, ce phénomène est aggravé par une création monétaire exceptionnelle destinée à financer l'effort de guerre. Il est toujours possible de drainer le pouvoir d'achat surnuméraire par des emprunts obligatoires, des impôts de guerre et un système de rationnement ${ }^{36}$. On ne peut guère compter que le gouvernement ivoirien des années 2000-2005 ait été en mesure, quelle qu'en soit la raison, de mettre en place de tels systèmes, de sorte que l'on peut s'attendre à ce que le taux d'inflation trahisse l'état de guerre. En fait, si des symptômes sont présents dans la série statistique proposée par la Banque mondiale ${ }^{37}$, ils sont modestes. Sur la période 2000-2012, le taux d'inflation moyen est de 2,9\% par an, or les chiffres pour 2002 et 2003 sont respectivement de 3,1 et 3,3. En revanche, et à nouveau, le chiffre de 2011 grimpe à 4,9\%, mais il est nettement surclassé par celui de 2008 à $6,3 \%$ (c'était $26 \%$ en 1994 et $27,4 \%$ en 1977 !). Pourtant l'examen de la création monétaire sur ces périodes montre bien que la circulation de monnaie, notamment manuelle, a considérablement augmenté en 2002 et 2003, grâce à des moyens mis à disposition par la Banque centrale des Etats de l'Afrique de l'ouest. Le chiffre de 2011 est sensiblement plus élevé mais ne détonne pas par rapport à la tendance des années 2006-2012.

\footnotetext{
36 John Maynard Keynes, «How to Pay for the War», Collected Writings of John Maynard Keynes, Londres, McMillan, 1978, tome XXII ; Jacques Aben, « D’Alger à Suez, Le Front républicain face au financement de ses guerres », Gestion et Finances Publiques-La Revue du Trésor, n ${ }^{\circ}$, 2012, pp.63-70

${ }^{37} \mathrm{http}$ ://donnees.banquemondiale.org/indicateur/FP.CPI.TOTL.ZG?locations=CI (consulté le 04/12/16). Il est à remarquer que dans le même temps l'INS-CI s'avère incapable de fournir de telles données.
} 


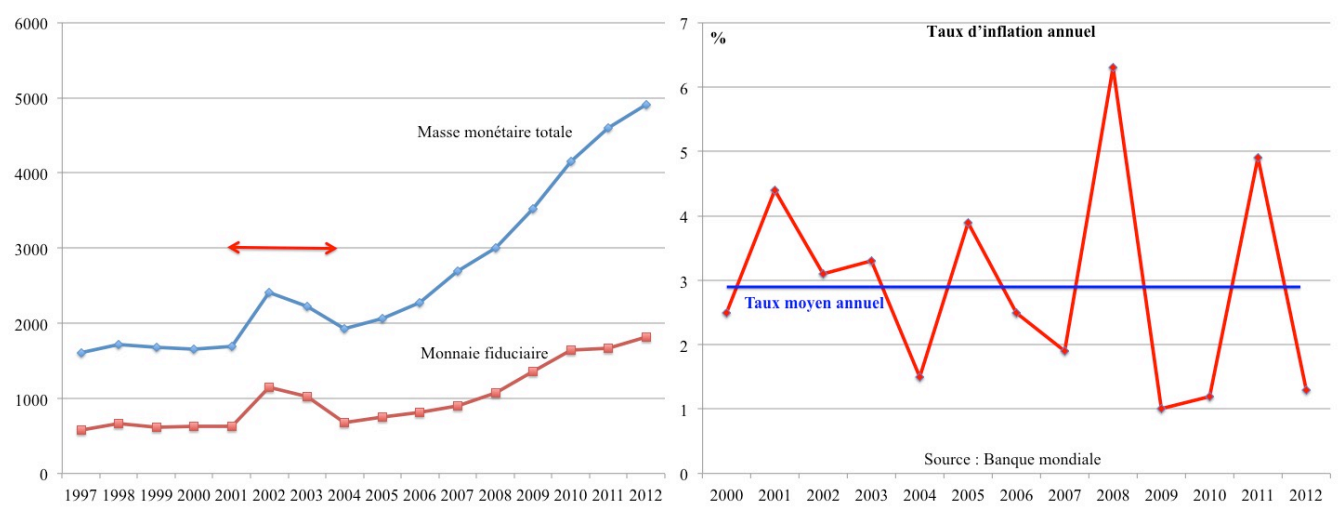

Pour en terminer avec cela, il est sans conteste possible de dire que l'économie de la Côte d'Ivoire a été marquée par la guerre civile, que ce soit au niveau de vécu quotidien ou dans ses variables macroéconomiques. Cela semble plus vrai pour la crise post-électorale que pour le début de la guerre, mais, encore une fois, cette période particulière se caractérise par une perte de contrôle par le gouvernement en place, sans que le président élu ait encore été en mesure de prendre les rennes du pouvoir. Toutes les énergies sont alors tournées vers la tentative de prise du pouvoir et l'activité économique peut attendre.

\section{3 - Une diplomatie de guerre}

La France, au travers notamment de son ambassadeur sur place, Renaud Vignal, a été la première à tenter une médiation entre le pouvoir légal et les mutins. Rien d'étonnant à cela vu l'influence de Paris dans les affaires de l'Afrique de l'ouest en général, de la Côte d'Ivoire en particulier, mais surtout vu les intérêts en jeu : 20000 expatriés français, de très nombreuses entreprises françaises présentes, souvent importantes (Bolloré, Orange, L'Oréal, Carrefour, Bel, par exemple), un fort risque de déstabilisation régionale. Donc, la position de la France à ce moment-là était fixée à la fois par certains principes généraux : souveraineté, stabilité et intégrité de la Côte d'Ivoire mais aussi des Etats environnants, tant les risques de transmission de la crise lui ont paru grands, eu égard à la porosité des frontières et à l'entremêlement des ethnies. Quelle qu'ait pu être l'opinion du gouvernement français sur la politique menée à Abidjan, il lui apparaissait clair qu'il ne fallait pas « laisser tomber » le gouvernement légal, quitte à le faire avec prudence pour ne pas rompre toute communication avec la rébellion. Ainsi Paris a accepté, dès le 28 septembre, de répondre positivement à la demande de matériels militaires formulée par la Côte d'Ivoire, tout en évitant d'envoyer des armements.

Néanmoins, souvent accusé de néocolonialisme, Paris souhaitait vivement que les Etats voisins soient les premiers acteurs de l'action diplomatique. Le 26 septembre, depuis le Maroc où séjournait le président du Gabon, Omar Bongo, un premier appel à une intermédiation africaine a été lancé. Le roi Mohamed VI a invité à Marrakech, outre Laurent Gbagbo, un certain nombre de membres de la Communauté économique des Etats de l'Afrique de l'ouest (CEDEAO) : Burkina Faso, Mali, Congo-Brazzaville, Togo, Sénégal, avec des représentants de l'Union africaine et de la France. Sachant la mauvaise qualité des relations entre la Côte d'Ivoire d'une part, le Burkina Faso et le Mali d'autre part, on comprend que Laurent Gbagbo ait souhaité que l'ensemble de la CEDEAO soit réuni, afin que ces deux pays voient leur influence réduite. En outre cela lui permettait de gagner du temps, à un moment où il pouvait croire que ses forces ne tarderaient pas à nettoyer les zones conquises par les rebelles.

C'est finalement le 29 septembre, à Accra (Ghana) donc en terrain neutre, que s'est tenue cette réunion plénière. Les principales propositions formulées ont été la désignation d'un groupe de contact assurant la liaison et l'organisation des négociations de paix entre les deux 
parties, ainsi que l'envoi sur place d'une force d'interposition, dont le mandat de serait pas de « réduire les rebelles» mais seulement « de paix et de bons officies », selon les termes du président en exercice, Abdoulaye Wade, président du Sénégal.

Il ne faudra qu'une semaine au groupe de contact pour obtenir l'accord de principe des deux parties pour un cessez-le-feu, qui aurait dû prendre effet le 7 octobre. Mais il faudra encore près de deux semaines de négociation au ministre des affaires étrangères sénégalais, Cheikh Tidiane Gadio, pour emporter la signature de Laurent Gbagbo qui aurait sans doute préféré que la CEDEAO vienne faire la guerre à ses côtés contre les mutins, plutôt que de le ravaler symboliquement au même niveau qu'eux.

Ce résultat heureux sera salué dès le 20 octobre, depuis Beyrouth, par la conférence au sommet de l'Organisation internationale de la Francophonie, mais avec une " déclaration » de nature à rendre les mutins soupçonneux, puisque ce texte condamnait "la tentative de prise de pouvoir par la force et la remise en cause de l'ordre constitutionnel en Côte d'Ivoire » ${ }^{38}$.

Néanmoins, les pourparlers de paix pouvaient commencer à Lomé, coordonnés par le président togolais Gnassimbé Eyadéma, désigné par la CEDEAO. Mais il n'a fallu que cinq semaines pour réaliser que cette médiation butait, d'une part sur la faible autorité de la CEDEAO et de ses négociateurs, d'autre part sur la mauvaise volonté des protagonistes de la crise, chacun devant penser qu'il pourrait finalement arriver à ses fins par la force. C'est pourquoi, dès le 10 décembre 2002, le ministre français des affaires étrangères pouvait annoncer la proposition de réunir à Paris les deux parties et les pays concernés, sous la présidence des autorités françaises. Et c'est justement Gnassingbé Eyadéma qui se chargera d'organiser une réunion préparatoire à Kara, au Togo, le 23 décembre.

Donc, "A l'invitation du Président de la République française » ${ }^{39}$, s'est tenue une tableronde des forces politiques ivoiriennes à Paris et à Linas-Marcoussis, entre le 15 et le 24 janvier 2003, sous la présidence ferme de Pierre Mazeaud, juriste reconnu et membre du Conseil Constitutionnel. C'est en effet la fermeté qui était à l'ordre du jour, comme l'avait annoncé le ministre des affaires étrangères lors des vœux à la presse le 14 janvier : "Nous devons aboutir - sinon - c'est le peuple ivoirien qui paiera ${ }^{40}$. Et accord il y a bien eu, qui a reçu l'onction de la Conférence des chefs d'Etat sur la Côte d'Ivoire, au centre Kléber, dès le 25 janvier. Cet accord marque une défaite pour Laurent Gbagbo, dans la mesure où, de manière claire, la légitimité des revendications de ceux qui devenaient les «ex-rebelles» étaient reconnue : un gouvernement «de réconciliation» sera mis en place dont le président de la république en exercice n'aura pas le contrôle de la composition ; ce gouvernement devra refonder une armée "attachée aux valeurs d'intégrité et de moralité républicaine »; il fera amnistier les militaires détenus pour atteinte à la sûreté de l'Etat; il reprendra la naturalisation des étrangers - «qui [ont] grandement contribué à la richesse nationale » - et modifiera les pratiques en matière d'identification des personnes; il fera modifier l'article 35 de la constitution fixant les conditions d'éligibilité du président de la république ${ }^{41}$.

Il n'est donc pas étonnant qu'après son retour à Abidjan, Laurent Gbagbo ait dit ses réserves à l'égard de cet accord et qu'une partie de la population d'Abidjan soit descendue dans la rue pour conspuer la France.

Il allait falloir des années de négociation pour que l'on finisse par aboutir à des élections, justement en application de l'accord de Marcoussis, et que s'ensuive la crise post-électorale. Entre 2003 et 2006, ces pourparlers entre pouvoir légal et ex-rebelles ont été menés

\footnotetext{
${ }^{38}$ http://www.francophonie.org/IMG/pdf/decl-beyrouth-2002.pdf, p.4, (consulté le 04/12/16)

39 http://www.diplomatie.gouv.fr/fr/dossiers-pays/cote-d-ivoire/colonne-droite/documents-de-reference/article/ accord-de-linas-marcoussis (consulté le 04/12/16).

${ }^{40}$ Le Monde, 15 janvier 2003.

${ }^{41}$ «Le candidat (...) doit être exclusivement de nationalité ivoirienne né de père ou de mère Ivoirien d'origine, au lieu de né ivoirien, de parents eux-mêmes ivoiriens. La guerre a peut-être tenu à cela.
} 
successivement par pas moins de douze négociateurs, sans compter la France et les organisations internationales ou régionales ${ }^{42}$. Bien sûr, la volonté des parties de ne pas aboutir à un accord ne satisfaisant pas leurs ambitions ou réduisant leur rente de « seigneurs de la guerre » aura été pour beaucoup dans ce marathon inefficace, d'autant qu'ils trouveront des partenaires dans d'autres pays : "'La réunion s'est très mal passée. On nous a fait tourner en bourriques depuis des jours', pestait dimanche soir [6 octobre 2002] un ministre des affaires étrangères du 'groupe de contact' de la CEDEAO. 'Il n'y a pas eu le moindre signe de bonne volonté' des autorités ivoiriennes ${ }^{43}$. Néanmoins il semble que les négociateurs eux-mêmes n'aient pas nécessairement été à la hauteur de la tâche, parfois emportés par la volonté d'être, et surtout de rester, LE négociateur: "le Président sénégalais Wade a opté, depuis son élection, pour la promotion médiatique de sa personne comme une star du show-biz (...) Nul n'a le droit de fossoyer les négociations de Lomé au nom d'un quelconque intérêt personnel. ${ }^{44}$

Après le «licenciement» du dernier médiateur africain, Thabo Mbéki, président de l'Afrique du Sud, par les Forces nouvelles, c'est la prise en main de la diplomatie par l'ONU qui fera sortir petit à petit le processus de paix de sa confortable léthargie. Ainsi, dans sa résolution 1657 du 6 février 2006, le Conseil de sécurité se déclare "gravement préoccupé par la persistance de la crise en Côte d'Ivoire et d'entraves de toutes parts au processus de paix et de réconciliation nationale », de sorte que le 7 février le Comité des sanctions sur la Côte d'Ivoire met en vigueur des sanctions individuelles - interdiction de voyager et gel des avoirs imposées à trois responsables ivoiriens accusés de menacer le processus de paix dans le pays, et prévues par la résolution 1572 du Conseil de sécurité : Charles Blé Goudé et Eugène Djué, deux dirigeants du mouvement des "Jeunes patriotes », partisans du président Gbagbo, et Laurent Gbagbo lui-même.

Si la résolution $1721 \mathrm{du} 1^{\mathrm{er}}$ novembre 2006 accepte la proposition de l'Union africaine de maintenir Laurent Gbagbo à la présidence de la république, du fait de l'impossibilité matérielle d'organiser les élections présidentielles maintes fois reportées, elle maintient aussi en place le premier ministre Charles Konan Banny, en lui donnant un mandat impératif visant à mener à bien toutes les tâches prévues par les accords de Marcoussis et d'aboutir à l'organisation des élections.

Finalement, le 4 mars 2007, Laurent Gbagbo et Guillaume Soro, son pire ennemi, signeront un accord faisant du second le premier ministre du premier, en remplacement de Konan Banny. Même si la route est encore pavée de nombre de chausse-trapes - Guillaume Soro échappe à un attentat à la roquette le 29 juin -, le nouveau premier ministre parviendra à organiser les élections présidentielles pour le 31 octobre 2010.

\section{4 - Licorne : une guerre française}

Le 19 septembre 2002, on peut imaginer que l'ambassadeur de France en Côte d'Ivoire, à Abidjan, le colonel commandant le $43^{\text {ème }}$ bataillon d'infanterie de marine à Port-Bouët et leurs correspondants respectifs au ministère des affaires étrangères et à l'état-major des armées ont immédiatement pensé aux 20000 ressortissants français expatriés en Côte d'Ivoire. En effet leur sauvegarde représentait, avec celle de leurs compatriotes dans les autres pays étrangers, l'un des "intérêts fondamentaux de la nation", tels que décrits par le code pénal en son article 410-1. Et parce qu'il s'agit d'intérêts fondamentaux, la France s'estime fondée à

\footnotetext{
${ }^{42}$ Yveline Dévérin, Crise ivoirienne : du bon usage de la médiation, http://www.pambazuka.org/fr (consulté le $4 / 12 / 16)$

${ }^{43}$ Le Monde, 7 octobre 2002.

${ }^{44}$ Le journal Le Matin, cité par Yveline Dévérin, op. cit.
} 
utiliser la force, si nécessaire, pour les défendre, au sens de feue l'ordonnance 59-147 du 7 janvier 1959.

Si nécessaire, mais aussi si possible. Les armées françaises sont toujours prêtes à intervenir immédiatement et loin. En permanence, plusieurs unités d'élite, plusieurs avions et navires sont en alerte dite " guépard », capables de partir en $12 \mathrm{~h}, 48 \mathrm{~h}$ ou $72 \mathrm{~h} .$. . Ainsi il est possible de lever rapidement jusqu'à l'effectif d'une demi-brigade $\left(2300^{45}\right)$, pour une opération interarmes, interarmées voire multinationale. A cet effet chaque brigade de l'armée de terre prend l'alerte pendant 6 mois.

C'est grâce à un tel système que la force Licorne a été initialement constituée et renforcée, des 500 pré-positionnés jusqu'au plus de 5000 mobilisés au plus fort de la crise, à la fin 2004 . Dès le début de 2003, avec un effectif de 2500, elle a pu être déconcentrée en 3 groupements tactiques interarmes (ouest, sud et centre) avec un GTIA spécifique de la zone d'Abidjan constitué autour du $43{ }^{\text {ème }}$ BIMA. Ce dispositif a encore été renforcé en 2004, par l'addition du bataillon de réserve opérationnelle associé à l'opération Corymbe (présence navale dans le golfe de Guinée) et constituant un GTIA temporaire (GTIAT).

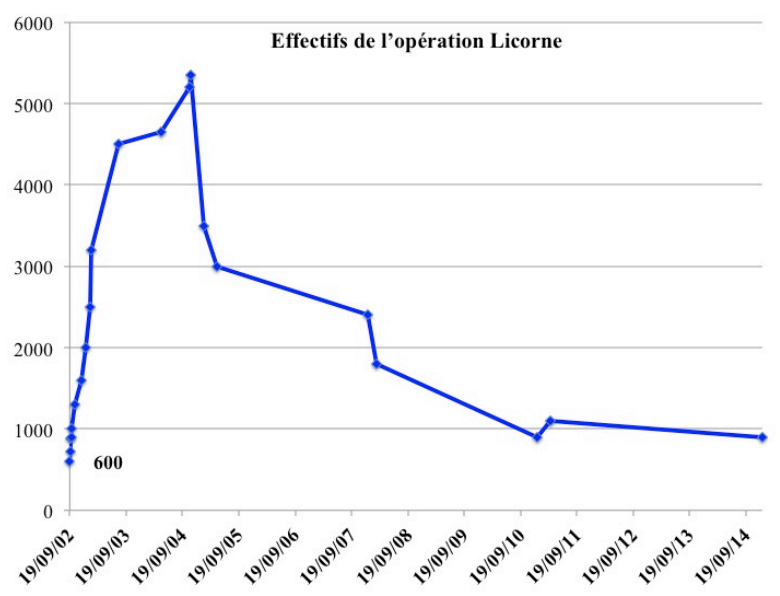

Si l'ensemble de la force était placé sous l'autorité d'un COMANFOR, du grade de général de division, celui-ci a été assisté au départ par un adjoint tactique, général de brigade, chargé de commander la manœuvre des unités de terrain par l'intermédiaire d'un état-major tactique (EMT). Plus tard (juillet 2003) cet officier général est devenu "adjoint opération », chargé d'assister le COMANFOR qui récupérait la pleine autorité sur la manœuvre des troupes via l'état major tactique, devenu entretemps " poste de commandement interarmées de théâtre » (PCIAT).

\footnotetext{
${ }^{45}$ Depuis le Livre blanc de 2013 , le contrat opérationnel prévoit une alerte permanente pour 5000 soldats, permettant de mettre en œuvre une force interarmées de réaction immédiate de 2300 soldats à $3000 \mathrm{~km}$ en 7 jours (Livre blanc, p.91)
} 


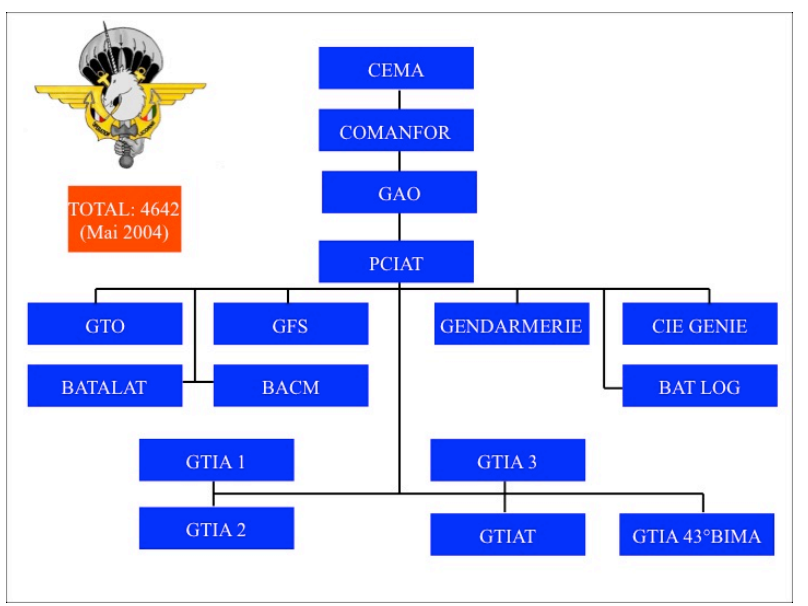

Entretenir une force de plusieurs milliers de militaires avec des centaines de véhicules de tous types et plusieurs aéronefs à plus de $4500 \mathrm{~km}$ de la métropole pose un problème logistique significatif, car les armées françaises ne possèdent pas en propre tous les moyens de transport nécessaires. Il faut donc procéder à la location de navires autant que d'avions pour assurer le soutien et les relèves quadrimestrielles. Mais cette location ne représente qu'une partie du supplément de coût que représente l'opération par rapport aux dépenses militaires courantes : avec les indemnités de sujétions pour service à l'étranger, le soutien vie et le soutien du matériel, Licorne a coûté 191 millions d'euros en $2004^{46}$.

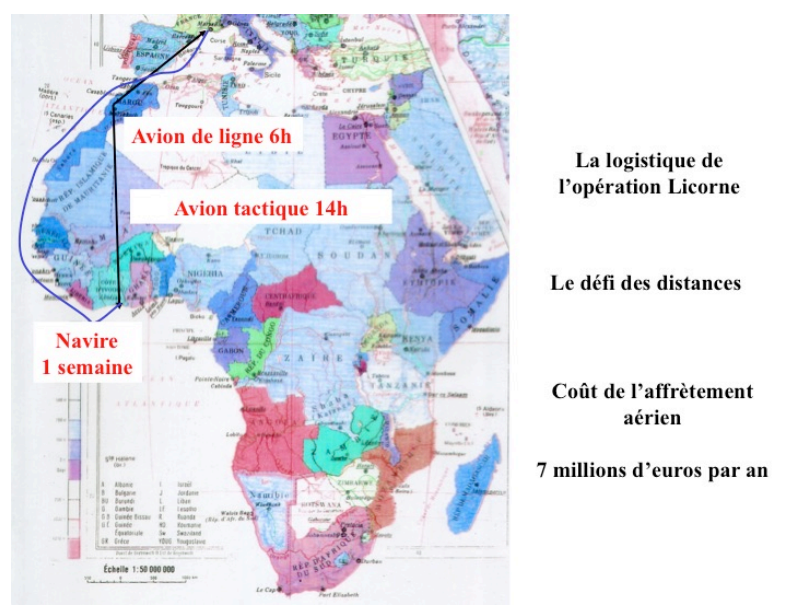

Lorsque les premiers renforts français sont arrivés à l'aéroport d'Abidjan, le dimanche 22 septembre 2002, la France n'avait reçu aucune demande officielle d'aide de la part du gouvernement légal ivoirien et ne bénéficiait d'aucun mandat du Conseil de sécurité des Nations Unies. Or ces forces, venues officiellement pour assurer la sécurité des expatriés, qu'ils soient ou non français, ont eu à forcer leur passage pour accomplir leur mission. Elles ont donc été, de fait, l'un des éléments du conflit armé, même si elles ont été placées sous des règles d'engagement très strictes, limitant l'ouverture du feu à la légitime défense élargie aux personnes à protéger. Le fait que le premier ministre ivoirien Pascal Affi N'Guessan ait indiqué à l'Agence France Presse que « «C'est tout à fait légitime. C'est le droit et c'est conforme aux accords que nous avons avec les pays européens qu'en cas de telle situation, ils prennent les dispositions pour assurer la sécurité de leurs ressortissants ${ }^{47}$ ne change rien à

\footnotetext{
46 Michelle Demessine et Jacques Peyrat, compte-rendu de mission en Côte d'Ivoire, https://www.senat.fr/rap/r08-178/r08-1784.html

${ }^{47}$ Le Monde, 22 septembre 2002.
} 
ce simple fait qu'une force armée étatique est venue s'ajouter aux belligérants déjà aux prises. De même, la demande d'aide officielle du gouvernement ivoirien intervenue entre le 27 et le 28 septembre 2002: "La Côte d'Ivoire a (...) actionné les accords de défense » ${ }^{48}$, n'a fait que donner une légalité internationale indirecte à l'intervention française, puisqu'il n'était question que de fourniture de moyens matériels, sans pour autant remettre en cause l'internationalisation.

La véritable légitimation, ou légalisation, de l'intervention française viendra avec la résolution 1464 du Conseil de Sécurité en date du 4 février 2003. En effet ce texte avalisait les accords de Linas-Marcoussis, donnant une fois de plus tort à Laurent Gbagbo, " se félicitait » du déploiement de la force de la CEDEAO et des troupes françaises pour contribuer à une solution pacifique à la crise, et «agissant en vertu du Chapitre VII de la Charte des Nations Unies », "autorise (...) les forces françaises (...) à prendre les mesures nécessaires pour assurer la sécurité et la liberté de circulation de leurs personnels et pour assurer, sans préjudice des responsabilités du Gouvernement de réconciliation nationale, la protection des civils immédiatement menacés de violences physiques à l'intérieur de leurs zones d'opérations et en fonction de leurs moyens ». C'est ce que l'on appelle un "mandat robuste » en langage diplomatique franglais. Il sera sans cesse renouvelé voire précisé et renforcé, comme avec la résolution 1528 du 27 février 2004, autorisant «les forces françaises, (...) à user de tous les moyens nécessaires pour (...) contribuer à la sécurité générale de la zone d'activité des forces internationales; (...) intervenir en cas d'éventuelles actions belligérantes, si les conditions de sécurité l'exigent, en dehors des zones placées sous le contrôle direct de l'ONUCI ; aider à protéger les civils dans les zones de déploiement de leurs unités ».

Et Licorne ne s'est pas privée d'utiliser cette autorisation tout au long de sa présence en Côte d'Ivoire, tout en mélangeant le mandat ainsi donné par le Conseil de Sécurité et celui, plus fluctuant, donné par le gouvernement français, en fonction de son analyse du rapport de forces et du rapport de légitimités entre Laurent Gbagbo et Guillaume Soro, ainsi que des intérêts de la France. En conséquence, Licorne a « fait la guerre » chaque fois que ses chefs, ou le chef des armées lui-même, l'ont jugé nécessaire ou souhaitable. La première mission, celle qui justifiait l'envoi de renforts au $43^{\text {ème }} \mathrm{BIMa}$, a été la sécurisation et, lorsque nécessaire, l'évacuation d'au moins 2200 ressortissants français et étrangers ${ }^{49}$ entre le 24 et le 29 septembre.

Le message accompagnant cette action était que Licorne constituerait aussi un "dispositif dissuasif », permettant d'éviter les évacuations. Mais il peut aussi être compris comme un moyen d'empêcher les mutins de descendre vers Abidjan et, profitant du capital de sympathie dont ils disposaient dans des quartiers comme Yopougon (1 à 2 millions d'habitants!), de renverser Laurent Gbagbo ${ }^{50}$. Ou encore, comme un moyen d'empêcher les FANCI de reconquérir le terrain perdu, d'où les manifestations furieuses et répétées des «jeunes patriotes » de Blé Goudé contre le camp du $43^{\text {ème }}$ BIMa.

Les opérations d'évacuation menées à Bouaké, Brobo, Ferkessédougou et Korhogo se sont faites sans usage des armes, parce que les mutins n'avaient aucune raison de s'y opposer. Curieusement, le premier échange de coups de feux s'est produit le 29 septembre à l'aéroport

\footnotetext{
${ }^{48}$ Pascal Affi N'Guessan cité par Le Monde du 28 septembre.

${ }^{49}$ Notamment 170 enfants américains d'une école chrétienne de Bouaké pris au piège des combats, et ce avec l'aide de 200 militaires américains des forces spéciales. Pour certains, la perspective inquiétante de voir conduire une telle opération seulement par des troupes américaines dans le cœur du pré carré français, aurait été l'élément déclencheur de la décision d'intervention du président Chirac.

${ }^{50}$ Dans les faits, il était clair en décembre 2002, que si les Français tiraient pour arrêter les rebelles, ils « levaient la barrière » pour permettre l'avancée des FANCI (Le Monde du 12/12/02 citant un officier français).
} 
de Korhogo, simplement parce que les factionnaires n'avaient pas été prévenus de l'atterrissage des hélicoptères français. Malgré des tirs d'armes lourdes, aucune perte n'a été enregistrée.

La manœuvre suivante, subie plutôt que voulue, a consisté à accepter "pour un temps limité », de garantir le cessez-le-feu du 17 octobre, dans l'attente que la CEDEAO mette en place sa propre mission d'interposition (MICECI). Présentée à l'époque comme secondaire par le général Beth, cette mission "d'observation et de sécurisation 》 assumée par Licorne devait en fait devenir essentielle, faisant des troupes françaises des «forces impartiales ». Pour ce faire, Licorne a continué de se renforcer (1200 hommes à la mi-octobre et 2500 à la fin de 2002) et a détaché des contingents à Brobo près de Bouaké, à l'est, Tiébissou, Daloa et Vavoua au centre et Man et Touba à l'ouest, créant ainsi une « ligne de non franchissement » (LNF) séparant les belligérants, mais dont l'efficacité n'a été que relative.

Le premier sang dans la confrontation entre Licorne et les forces en présence, a été tiré le $1^{\text {er }}$ décembre lors de la sécurisation de l'aéroport de Man, préparatoire à l'évacuation de 160 personnes : un blessé français pour huit rebelles tués.

Les accrochages se sont multipliés à partir du 28 novembre avec l'entrée en guerre du MPIGO et du MJP, mais les hélicoptères français n'ont pas dissuadé les Mi24 FANCI, pilotés par des mercenaires biélorusses, d'attaquer des positions rebelles au nord de la LNF, devenue entre-temps ligne de cessez-le-feu (LCF).

Ce n'est qu'au mois de mars 2003 que la MICECI viendra prendre sa part de la mission d'interposition en plaçant 900 de ses 1400 soldats en premier échelon le long de la LCF. Licorne, érigée en force de réaction rapide par la résolution 1464, en assurera le soutien. Le premier acte important mis en œuvre par le duo MICECI-Licorne sera de créer, en coopération avec les FANCI et les FAFN, une première zone de confiance de $40 \mathrm{~km}$ sur 60 dans le grand ouest, afin de mettre fin aux violences et aux exactions commises par des irréguliers. Cette zone servira en quelque sorte d'expérimentation pour créer, en juin 2003, la zone de confiance (ZDC) qui, de Zouan Hounien au sud-ouest à Bania au nord-est, prendra en écharpe la Côte d'Ivoire jusqu'en avril 2007. Au centre de cette zone, le 25 août, Licorne subira ses deux premières pertes au combat, par des tirs irrationnels de rebelles avinés.

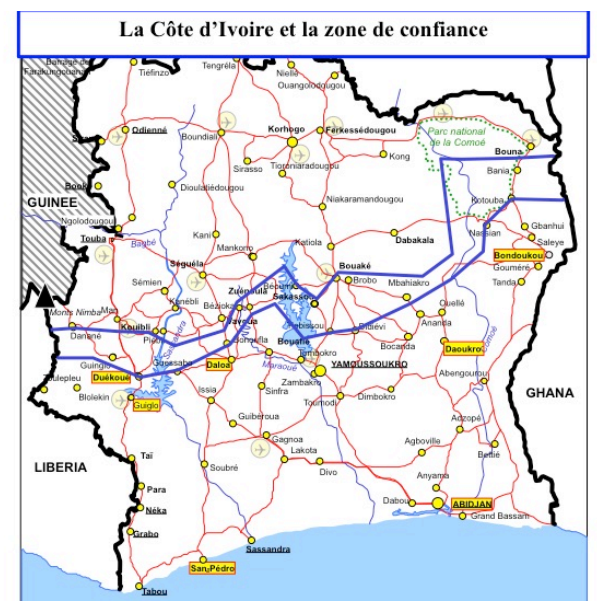

Avec la résolution 1479 du 13 mai 2003, Licorne se voit demander d'appuyer le processus de désarmement, démobilisation, réinsertion (DDR) ${ }^{51}$, prévu par les accords de Linas-Marcoussis et que l'ONU doit lancer. Il s'agit de trouver les combattants, de leur racheter leurs armes et de les préparer à un retour à la vie civile et à un emploi. Sauf qu'il ne démarrera qu'en 2013.

\footnotetext{
${ }^{51} \mathrm{Ou}$ « Désarmement, démobilisation, réinsertion, rapatriement et réinstallation », selon les termes de la résolution 1528 .
} 
En attendant, c'est Licorne qui va occuper le terrain par son bureau des actions civilomilitaires (BACM). A côté des Tactical support teams (TST) qui sont l'instrument habituel des actions civilo-militaires, le BACM mettra en place des «équipes de chantier» dans les régions de Bouaké, Man et Danané, afin de lancer des projets d'intérêt général susceptibles de mobiliser les combattants, évitant ainsi qu'ils soient réduits à devenir des coupeurs de route. Ce sera par exemple le cas à Daloa, où les soldats des FAFN rétabliront le circuit d'eau courante dans l'hôpital.

Pour Licorne, l'année 2004 représente un point culminant de la violence, puisque le 6 novembre les deux Sukhoi 25 des FANCI qui avaient bombardé les lignes FAFN la veille et l'avant-veille, viendront bombarder le cantonnement du GTIA1 à Bouaké, faisant 9 morts et 37 blessés parmi les militaires français. Les représailles ne se sont pas fait attendre, puisque le président de la république, chef des armées, a ordonné la destruction de tous les aéronefs susceptibles de s'en prendre à nouveau aux troupes françaises. La réaction en chaîne qui s'en est suivie n'avait probablement pas été prévue par les planificateurs de l'état-major des armées : des milliers d'Abidjanais dans la rue hurlant contre Licorne et menaçant les expatriés ; des tirs de dissuasion pour arrêter la foule au niveau des ponts Houphouët-Boigny et Charles-de-Gaulle ; une section égarée à l'hôtel Ivoire et livrée à la foule par les gendarmes ivoiriens ; au moins une soixantaine de tués imputés à Licorne, qui n'en reconnaît que 20.

Le dernier acte important de l'opération Licorne interviendra en avril 2011, en pleine crise postélectorale, à la demande de l'ONU, pour empêcher les FANCI et les milices pro-Gbagbo de commettre des exactions contre la population des quartiers d'Abidjan réputés pro-Ouattara. Là encore le feu a été ouvert par tous les moyens disponibles depuis le fusil d'assaut jusqu'au missile anti-char porté par hélicoptère. En appuyant indirectement les FAFN, Licorne permettra à celles-ci de prendre le dessus, de ramener le calme dans Abidjan et finalement d'installer Alassane Ouattara à la présidence de la Côte d'Ivoire, en application des résultats des élections reconnus par l'ONU - un beau renversement par rapport à «l'ouverture des barrières » de 2002.

\section{Conclusion}

Ainsi Licorne s'est retrouvée au milieu d'une «guerre civile» qui aura fait des milliers de morts et occasionné des comportements justiciables de la Cour pénale internationale. Mandatée par le seul Etat français ou par le Conseil de sécurité, elle a usé de la force létale chaque fois que les militaires y ont été contraints par leurs adversaires, ou que cela leur a été commandé, sans que l'on sache vraiment, à l'issue du conflit, combien de pertes son action a occasionnées : «Les versions divergent sur le nombre de victimes côté mutins (...) Dans les rangs français, il n'y avait pas eu de victimes, tandis qu'aucun chiffre n'était disponible chez les rebelles $»{ }^{52}$. Et à côté de cela, combien de fois les soldats de Licorne se sont-ils trouvés «à deux doigts de déclencher un premier tir de sommation » ${ }^{53}$, en ayant la maturité et la formation voulue pour ne pas transgresser le droit de la guerre ni perdre leur sang-froid ? Euxaussi ont payé le prix du sang, même s'il peut paraître faible : 27 morts, dont seulement 11 " pour la France » ${ }^{54}$, car il y a maintes manières de mourir en opération ${ }^{55}$.

La guerre civile est donc devenue une guerre internationale par la présence d'un autre Etat que la Côte d'Ivoire, surtout dans la mesure où cet autre Etat est intervenu de sa propre initiative, pour défendre ses intérêts fondamentaux. C'est d'autant plus vrai qu'au delà de la

\footnotetext{
${ }^{52}$ Le Monde des 23 et 29 décembre 2002.

${ }^{53}$ Propos d'un officier de « marsouins » rapportés par Le Monde du 5 février 2004.

${ }^{54}$ Rapport du groupe de travail « Monument aux morts en opérations extérieures », Paris, 2011, p.51.

${ }^{55} \mathrm{Si}$ un soldat qui attendait à l'arrière d'une $\mathrm{P} 4$ à un point de contrôle FANCI n'avait pas eu l'idée de changer de côté sur sa banquette, il n'aurait peut-être pas reçu la balle de 7,62 tirée accidentellement par un factionnaire.
} 
protection de ses ressortissants, il a pu peser sur l'évolution du conflit successivement au profit de chacune des parties. Bien sûr l'internationalisation a encore été accrue par l'intervention militaire de la CEDEAO d'abord, des Nations Unies ensuite.

Alors serait-il abusif d'employer le mot « guerre » pour traiter de l'engagement de Licorne en Côte d'Ivoire?

C'est finalement le ministère de la défense qui rechigne à aller jusque là, quelles que soient ses raisons. Le premier symptôme se trouve dans le fait que même si des militaires ayant servi en Côte d'Ivoire se sont vus reconnaître le statut de « combattant » pour avoir participé à des actions de combat ou avoir appartenu à des unités combattantes, aucun ne s'est vu attribuer le régime de la « campagne double », à la différence de leurs camarades engagés en République centrafricaine qui ont été " exposés à des situations de combat» ${ }^{56}$. Le second symptôme concerne la non attribution de la " croix de guerre des théâtres d'opération extérieurs » à des combattants de Licorne qui auraient obtenu des citations individuelles «pour services de guerre caractérisés directement liés à l'intervention ». Mais il faut dire que le dernier théâtre ayant bénéficié de cette attribution est le Kosovo, pour les seules actions intervenues entre le 24 mars et le 21 juin 1999, même si elles n'ont occasionné aucune perte aux militaires français.

Alors, guerre, pas guerre?

\footnotetext{
${ }^{56}$ Décret $\mathrm{n}^{\circ}$ 2015-947 du 31 juillet 2015. «Les bénéfices de campagne sont des bonifications qui s'ajoutent, dans le décompte des trimestres liquidés, à des périodes de services militaires ou assimilées à des services militaires », https://www.cdc.retraites.fr/portail/spip.php?page=article\&id_article=8986\&cible=_employeur.
} 